\title{
Neoadjuvant intra-arterial chemotherapy using an original four- lumen double-balloon catheter for locally advanced uterine cervical cancer
}

\author{
Tomohito Tanaka ${ }^{1}$, Yoshito Terai ${ }^{1}$, Satoe Fujiwara ${ }^{1}$, Yoshimichi Tanaka ${ }^{1}$, Hiroshi \\ Sasaki ${ }^{1}$, Satoshi Tsunetoh ${ }^{1}$, Kazuhiro Yamamoto ${ }^{2}$, Takashi Yamada ${ }^{3}$ and Masahide \\ Ohmichi $^{1}$ \\ ${ }^{1}$ Department of Obstetrics and Gynecology, Osaka Medical College, Takatsuki, Japan \\ ${ }^{2}$ Department of Radiology, Osaka Medical College, Takatsuki, Japan \\ ${ }^{3}$ Department of Pathology, Osaka Medical College, Takatsuki, Japan \\ Correspondence to: Yoshito Terai, email: y-terai@osaka-med.ac.jp \\ Keywords: uterine cervical cancer; locally advanced uterine cervical cancer; neoadjuvant chemotherapy; radical hysterectomy \\ Received: June 05, $2018 \quad$ Accepted: December 13,2018 Published: December 28, 2018 \\ Copyright: Tanaka et al. This is an open-access article distributed under the terms of the Creative Commons Attribution License \\ 3.0 (CC BY 3.0), which permits unrestricted use, distribution, and reproduction in any medium, provided the original author and \\ source are credited.
}

\section{ABSTRACT}

OBJECTIVE: We report a balloon-occluded arterial infusion therapy with an original four-lumen double-balloon catheter (4L-DB) which allows for the efficient injection of an anticancer agent at a high concentration to the target spot for patients with locally advanced uterine cervical cancer.

METHODS: One hundred and forty-three patients with locally advanced cervical cancer treated with neoadjuvant intra-arterial chemotherapy (NAIAC) or a primary radical hysterectomy (PRH) were retrospectively assessed. The patients in the NAIAC group received irinotecan $70 \mathrm{mg} / \mathrm{m} 2$ intravenously on day 1 and 8 and cisplatin 70 $\mathrm{mg} / \mathrm{m} 2$ intra-arterially using the 4L-DB on day 2 of a 21-day course, and two courses were performed in principle. The radical hysterectomy was performed within 6 weeks after NAIAC.

RESULTS: Ninety-four patients were treated with NAIAC, and 49 patients undertook a PRH. The response rate of NAIAC on MRI was $\mathbf{9 2 . 6 \%}$. Fourteen patients $(\mathbf{1 4 . 6 \% )}$ had no evidence of cancer cells on pathologic diagnoses. The NAIAC group had a longer disease-free survival than the PRH group $(p=0.02)$; however, the overall survival was not significantly different. The relative risk (RR) for recurrence was higher in patients with lymph node metastasis ( $R R, 4.31 ; 95 \% \mathrm{CI}, 2.23-8.43$ ) and lower in those who underwent NAIAC (RR, 0.30; 95\% CI, 0.14-0.68).

CONCLUSION: Our results with NAIAC using the 4L-DB catheter in locally advanced cervical cancer indicates beneficial effects on primary lesions and improves disease-free survival.

\section{INTRODUCTION}

We previously reported a neoadjuvant intra-arterial chemotherapy (NAIAC) using an original four-lumen double-balloon (4L-DB) catheter (Figure 1) [1]. This catheter can expand the balloon in the internal iliac artery on the central and peripheral side of the uterine artery and selectively inject the anticancer agent into the uterine artery; the anticancer drug will not flow into other arteries, such as the inferior vesical artery, middle rectal artery, inferior gluteal artery or obturator artery. Therefore, a high concentration of the anticancer drug can be directly delivered to tumors through the uterine artery. The 4L-DB has also been used for bladder cancer [2-8], as well as cervical cancer [1]. Women with locally advanced cervical cancer (stage IB2, IIA2 and IIB) 
have a higher rate of recurrence and poor survival than those with early-stage disease (stage IA, IB1 and IIA1) [9]. In accord with the National Comprehensive Cancer Network's (NCCN) clinical guidelines, surgery or concurrent chemoradiotherapy (CCRT) is recommended in patients with stage IB1 and, in IIA1 cases, neoadjuvant chemotherapy (NAC) is not recommended [10] because a meta-analysis showed no advantage for NAC [11]. For patients with stage IB2, IIA2 and IIB disease, CCRT is also recommended, especially in patients with lymph node metastasis [10]. However, a NAC followed by radical hysterectomy is often commented on because several studies, including meta-analysis, have shown that NAC may improve the patient's prognosis $[12,13]$. However, the appropriate treatment of stage IB2, IIA2 and IIB remains uncertain.

Adenocarcinoma currently comprises $10-20 \%$ of all cervical carcinomas in developed countries, compared to $5-10 \%$ three decades ago [14], and has a worse prognosis than squamous cell carcinoma $[15,16]$. Several studies have shown that a radical hysterectomy has a better prognosis than radiotherapy in cervical adenocarcinoma $[9,17]$. However, it may be difficult to perform a radical hysterectomy because cervical adenocarcinomas tend to form bulky tumors in some cases. NAC followed by a radical hysterectomy may improve the prognosis for bulky cervical adenocarcinoma. Cisplatin has been a key drug for cervical cancer because it has a $20 \%$ to $30 \%$ response rate as a single agent [18]. Irinotecan also has anticancer activity on cervical adenocarcinomas [19]. The response rate of intravenous neoadjuvant chemotherapy with cisplatin and irinotecan or nedaplatin and irinotecan for cervical cancer is $78.0-89.5 \%$ [20-23].

The current study demonstrates the efficacy and tolerance of NAIAC with cisplatin and irinotecan using a 4L-DB in patients with locally advanced cervical cancer, including adenocarcinoma.

\section{RESULTS}

Among the 143 patients in the study with locally advanced cervical cancer, 94 patients received NAIAC, and 49 patients underwent primary radical hysterectomy (PRH) (Figure 2). Among the 94 patients who received NAIAC, 88 patients received two courses of NAIAC. The other 6 patients received one course of NAIAC because two patients had progression of the disease, one patient had no tumor regression, one patient had serious neuropathy,

\section{BOAI System}

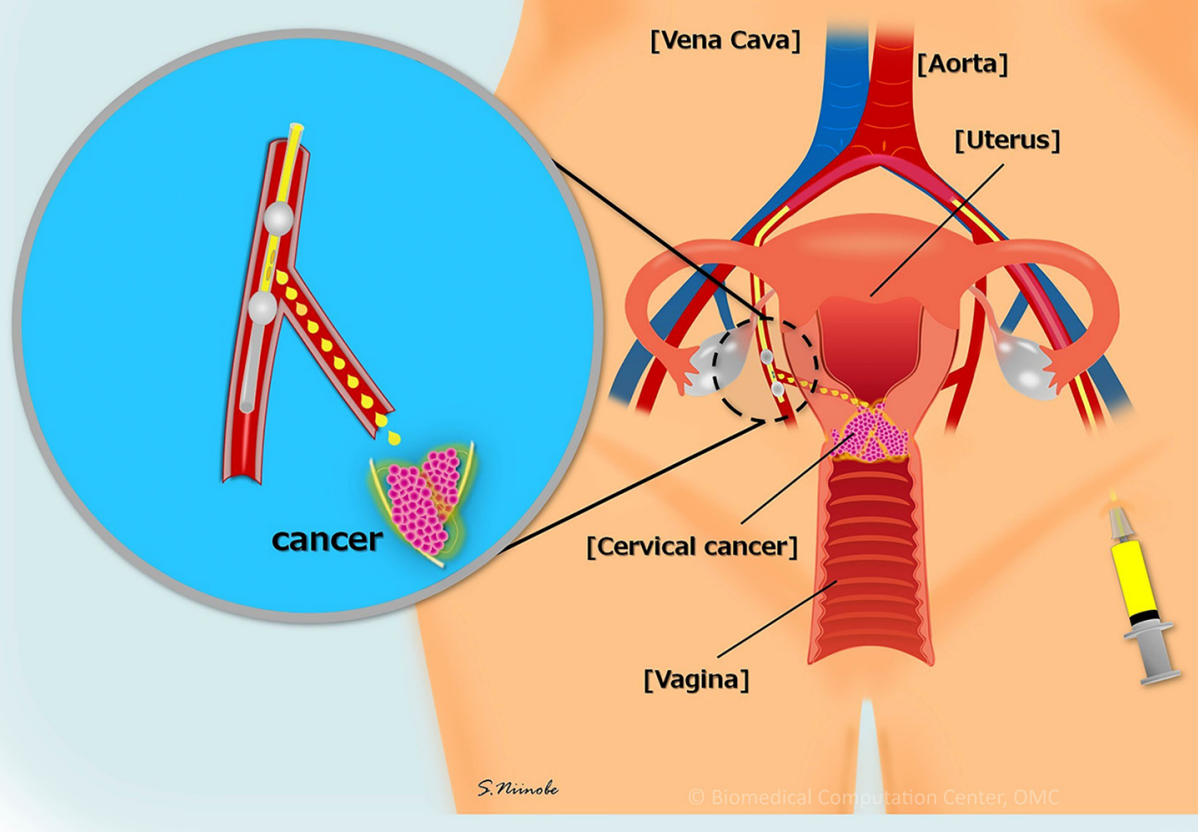

Figure 1: Balloon-occluded arterial infusion therapy (BOAI). Original four-lumen double-balloon (4L-DB) catheter has a double balloon on the end. A slit between the double balloon allows for the infusion of the anticancer drug locally. The catheter is inserted from the femoral artery. Through the opposite internal iliac artery, the end is placed on the superior gluteal artery. The anticancer drug injected through the slit remains between the double balloon and is delivered to the uterine artery or feeding artery selectively. 
and one patient had general fatigue. Among the 88 patients who received two courses of NAIAC, 85 patients underwent radical hysterectomy $(\mathrm{RH})$ after NAIAC. The other 3 patients received CCRT after NAIAC because one patient had progression of the disease, one patient had no tumor regression, and one patient experienced serious weight loss. Among the six patients who received one course of NAIAC, three underwent $\mathrm{RH}$, and three received CCRT after NAIAC (Figure 1). Table 1 shows the characteristics, treatment and side effects of the 94 patients with locally advanced cervical cancer who underwent NAIAC. The mean ( \pm standard deviation, SD) age of the patients was $49.4 \pm 11.4$ years. A total of 13 patients had the International Federation of Obstetricians and Gynecologists (FIGO) stage IB2 disease, 17 had stage IIA2 disease, and 64 had stage IIB disease. Histologically, 73 patients had squamous cell carcinoma, and 21 had adenocarcinoma. Eighty-eight patients underwent two courses of NAIAC, and the other six patients had only one course of NAIAC. Eighty-eight patients underwent RH after NAIAC and the other six patients received CCRT after NAIAC. The mean $( \pm \mathrm{SD})$ tumor size was $46 \pm 13 \mathrm{~mm}$ before NAIAC, decreasing to $14 \pm 16 \mathrm{~mm}$ after NAIAC. The most frequent side effect was blood toxicity. Forty- six patients $(48.9 \%)$ had grade 3 or grade 4 leukopenia. One patient $(1.1 \%)$ had grade 3 thrombocytopenia. One $(1.1 \%)$ had grade 3 anemia. There were no other grade 3 or more side effects. Table 2 shows the response to NAIAC for magnetic resonance imaging (MRI) and pathology. Among the 94 patients with locally advanced cervical cancer who underwent NAIAC, 37 patients had complete response (CR), 50 patients had partial response (PR), four patients had stable disease (SD), and three patients had progressive disease (PD) for MRI, resulting in a response rate of $92.6 \%$. Among the 73 patients with squamous cell carcinoma who underwent NAIAC, 29 patients had CR, 39 patients had $\mathrm{PR}$, three patients had SD, and two patients had PD, thus yielding a response rate of $93.2 \%$. Among the 21 patients with adenocarcinoma who underwent NAIAC, eight patients had CR, 11 patients had PR, one patient had $\mathrm{SD}$, and one patient had $\mathrm{PD}$, resulting in a response rate of $90.5 \%$. Pathologically, among the 88 patients with locally advanced cervical cancer who underwent NAIAC followed by $\mathrm{RH}, 14$ patients $(15.9 \%)$ had grade 3 response, 41 patients had grade 2 response, 23 had grade $1 \mathrm{~b}$ response, 9 had grade 1a response, and one patient had grade 0 response. Among the 68 patients with squamous cell carcinoma, 13 patients (19.1\%) had grade 3 response, 31

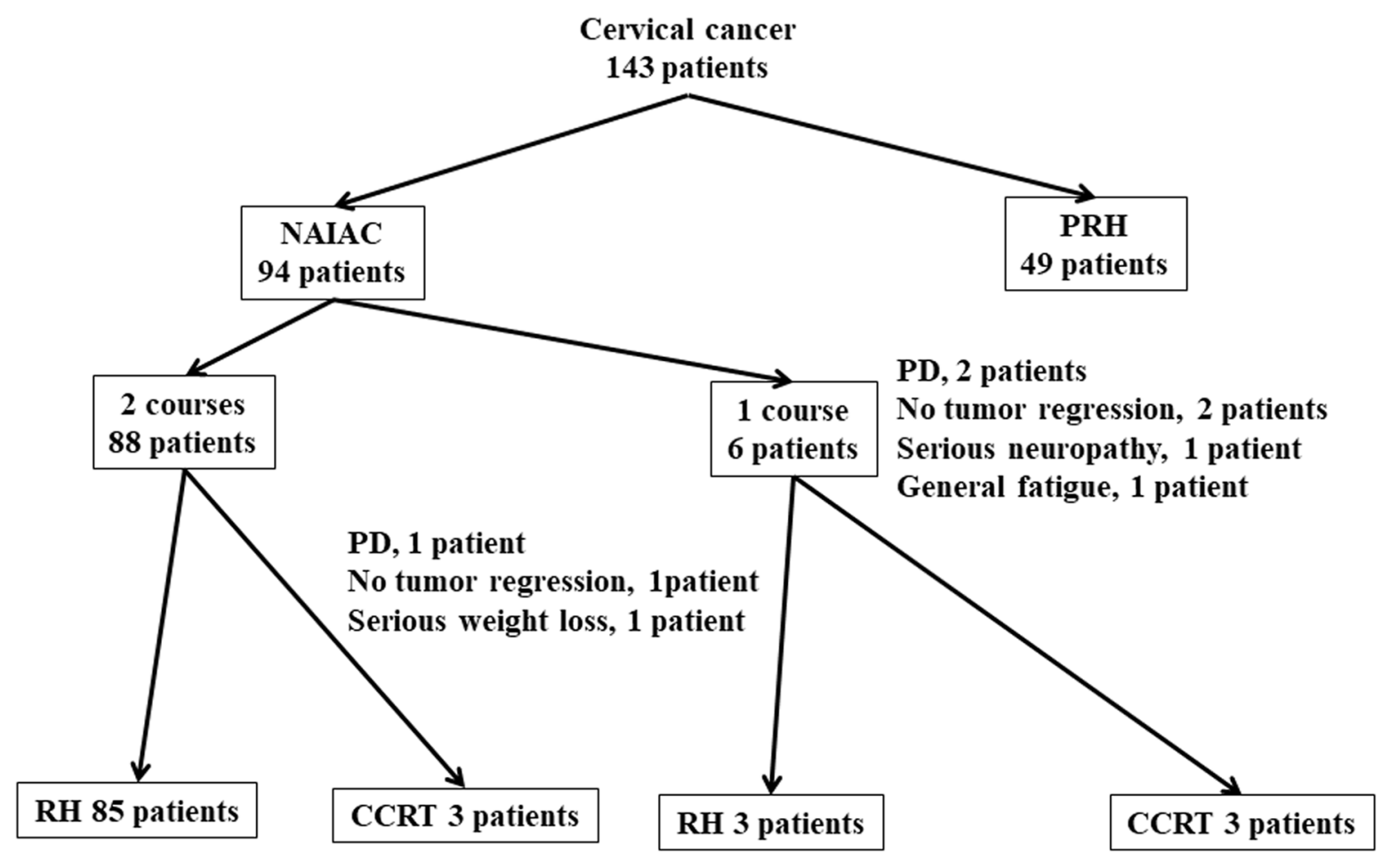

Figure 2: Among the 143 patients with locally advanced cervical cancer, 94 patients received neoadjuvant intraarterial chemotherapy (NAIAC), and 49 patients underwent primary radical hysterectomy (PRH). Among the 94 patients who receive NAIAC, 88 patients receive two courses of NAIAC. The other 6 patients receive one course of NAIAC because two patients had progressive disease (PD), one patient had no tumor regression, one patient had serious neuropathy, and one patient had general fatigue. Among the 88 patients who received two courses of NAIAC, 85 patients underwent radical hysterectomy (RH) after NAIAC. The other three patients receive CCRT after NAIAC because one patient had progression of the disease, one patient had no tumor regression, and one patient experienced serious weight loss. Among the six patients who received one course of NAIAC, three underwent RH, and three receive CCRT after NAIAC. 
Table 1: Patients with locally advanced cervical cancer who received NAIAC

\begin{tabular}{|c|c|}
\hline Total number of patients & 94 \\
\hline Age (years old) & $49.4 \pm 11.4$ \\
\hline \multicolumn{2}{|l|}{ FIGO stage } \\
\hline IB2 & 13 \\
\hline IIA2 & 17 \\
\hline IIB & 64 \\
\hline \multicolumn{2}{|l|}{ Histology } \\
\hline Squamous cell carcinoma & 73 \\
\hline Adenocarcinoma & 21 \\
\hline \multicolumn{2}{|l|}{ NAIAC } \\
\hline Two courses & 88 \\
\hline One course & 6 \\
\hline \multicolumn{2}{|l|}{ Treatment after chemotherapy } \\
\hline Radical hysterectomy & 88 \\
\hline CCRT & 6 \\
\hline \multicolumn{2}{|l|}{ Tumor size } \\
\hline Before NAIAC (mm) & $46 \pm 13$ \\
\hline After NAIAC (mm) & $14 \pm 16$ \\
\hline \multicolumn{2}{|l|}{ Side effect (grade 3 or more) } \\
\hline Leukopenia & 46 \\
\hline Thrombocytopenia & 1 \\
\hline Anemia & 7 \\
\hline
\end{tabular}

NAIAC, neoadjuvant intra-arterial chemotherapy; FIGO, The International Federation of Obstetricians and Gynecologists; CCRT, concurrent chemoradiotherapy.

patients had grade 2 response, 18 had grade $1 \mathrm{~b}$ response, five had grade 1a response and one patient had grade 0 response. Among the 20 patients with adenocarcinoma, one patient $(5.0 \%)$ had grade 3 response, 10 patients had grade 2 response, five had grade $1 \mathrm{~b}$ response, and four had grade 1a response. Table 3 shows the characteristics of patients who underwent NAIAC followed by RH and primary RH. Eighty-eight patients underwent NAIAC followed by RH. In contrast, 49 patients underwent primary RH. The mean ( \pm standard deviation, SD) age was not significantly different between the groups $(49.0 \pm 11.4$ vs. $50.5 \pm$ 11.5 years, $p=0.9)$. In the NAIAC group, 12 patients had FIGO stage IB2 disease, 17 had stage IIA2 disease, and 59 had stage IIB disease. In the PRH group, 15 patients had FIGO stage IB2 disease, 17 had stage IIA2 disease and 17 had stage IIB disease; the rate of IIB disease was significantly higher in the NAIAC group than that in the PRH group $(67.0 \%$ vs. $34.7 \%, p<0.01)$. Histologically, $68(77.3 \%)$ patients had squamous cell carcinoma, and $20(22.7 \%)$ had adenocarcinoma in the NAIAC group. In contrast, 28 (57.1\%) patients had squamous cell carcinoma, and 21 (42.9\%) had adenocarcinoma in the PRH group; the rate of adenocarcinoma was significantly lower in the NAIAC group than that in the PRH group $(22.7 \%$ vs. $42.9 \%, p=0.04)$. The mean pretreatment tumor size for MRI was not significantly different between the two groups ( $45 \pm 12$ vs. $44 \pm 14 \mathrm{~mm}, \mathrm{p}=0.6)$. In the NAIAC group, 27 patients underwent RT or CCRT, and 30 patients underwent chemotherapy after RH. The other 31 patients did not undergo adjuvant therapy. In the PRH group, 25 patients underwent RT or CCRT, and 19 patients underwent chemotherapy after RH. The other five patients did not undergo adjuvant therapy; the rate of no adjuvant therapy was lower in the NAIAC group than that in the PRH group $(35.2 \%$ vs. $10.2 \%, \mathrm{p}<0.01)$. One of 31 patients with no adjuvant therapy in the NAIAC group experienced recurrence on the vaginal stump. In contrast, two of five patients with no adjuvant therapy in the PR group had recurrence in the lung and pelvic cavity. Median follow up was shorter in the NAIAC group than that in the PRH group ( 30 vs. 48 months, $\mathrm{p}<0.01$ ). The three-year disease free survival rate was significantly higher in the NAIAC 
Table 2: Response to NAIAC

\begin{tabular}{|c|c|c|c|c|c|c|c|c|c|c|c|c|}
\hline \multicolumn{7}{|c|}{ Response to NAIAC } & \multicolumn{6}{|c|}{ Response to NAIAC followed by RH } \\
\hline & \multirow{2}{*}{$\begin{array}{c}\text { Number } \\
\text { of patients }\end{array}$} & \multicolumn{4}{|c|}{ Response for MRI } & \multirow[t]{2}{*}{$\mathbf{R R}$} & \multirow{2}{*}{$\begin{array}{c}\text { Number } \\
\text { of patients }\end{array}$} & \multicolumn{5}{|c|}{ Pathological response } \\
\hline & & CR & PR & SD & PD & & & $\mathbf{0}$ & $1 \mathbf{a}$ & $1 b$ & 2 & 3 \\
\hline Total & 94 & 37 & 50 & 4 & 3 & 92.6 & 88 & 1 & 9 & 23 & 41 & 14 \\
\hline \multicolumn{13}{|c|}{$\begin{array}{l}\text { Histological } \\
\text { type }\end{array}$} \\
\hline $\mathrm{SCC}$ & 73 & 29 & 39 & 3 & 2 & 93.2 & 68 & 1 & 5 & 18 & 31 & 13 \\
\hline $\mathrm{AD}$ & 21 & 8 & 11 & 1 & 1 & 90.5 & 20 & 0 & 4 & 5 & 10 & 1 \\
\hline
\end{tabular}

NAIAC, neoadjuvant intra-arterial chemotherapy; RH, radical hysterectomy; MRI, magnetic resonance imaging; SCC, squamous cell carcinoma; AD, adenocarcinoma; CR, complete response; PR, partial response; SD, stable disease; PD, progressive disease.

group than that in the PRH group (64\% vs. $52 \%, \mathrm{p}=0.02)$. Figure 3 shows the disease free survival rate and overall survival rate for both groups. Those patients in the NAIAC group had a longer disease free survival than those in the PRH group ( $p=0.02)$. However, overall survival was not significantly different between the two groups. Figure 4 shows the results of multivariant analysis for the risk of recurrence. Lymph node metastasis (RR, 4.31; 95\%CI, 2.23-8.43) and NAIAC (RR, 0.30; 95\%CI 0.14-0.68) were independent factors for recurrence. Other factors, including histological type, lymphovascular involvement, deep stromal invasion (more than half myometrial invasion), bulky tumor (more than $4 \mathrm{~cm}$ ), positive cut end and parametrial invasion, were not independent risk factors on multivariant analysis. Statistically, parametrial invasion was not an independent factor for recurrence (RR, 0.40; 95\% CI, 0.14-1.02); however, this result suggested that those patients with parametrial invasion were less likely to experience recurrence. Most patients with risk factors including lymph node metastasis, LVI, deep stromal invasion, bulky tumor, positive cut end, and parametrial invasion received adjuvant chemotherapy or radiotherapy. Most patients with parametrial invasion received adjuvant radiotherapy, thus adjuvant radiotherapy may bring better DFS.

\section{DISCUSSION}

NAIAC using a 4L-DB with intravenous irinotecan and intra-arterial cisplatin was feasible and effective on locally advanced cervical adenocarcinoma as well as squamous cell carcinoma. The NAIAC group had excellent DFS, compared with the PRH group. However, overall survival was not significantly different between the two groups. Moreover, the NAIAC group required less frequent adjuvant therapy than the PRH group.

The 4L-DB is an original catheter for intra-arterial chemotherapy which allows for a high concentration anticancer drug to be directly delivered to tumors through the uterine artery [1]. We previously reported NAIAC using a 4L-DB with platinum, mitomycin, and pirarubicin for cervical squamous cell carcinomas. The results revealed a $96.7 \%$ response rate with a $20 \%$ pathological CR [1]. The current study revealed a $93.2 \%$ RR with a $19.1 \%$ pathological CR for squamous cell carcinomas and a $90.5 \%$ PR with a $5 \%$ pathological CR for adenocarcinomas. The 4L-DB has been used not only for uterine cervical cancer but also for bladder cancer [2-8]. The Bladder preservation therapy combining intraarterial chemotherapy using a 4L-DB and radiotherapy has already been performed.

There have been several literatures about NAC with platinum and CPT-11 for treatment of cervical cancer. Sugiyama et al. reported about NAC followed by a radical hysterectomy for stage of IB2 to IIIB cervical cancer. In this report, 23 patients were treated with intravenous CPT-11 (60 mg/m2, day 1,8 and 15) and cisplatin (60 $\mathrm{mg} / \mathrm{m} 2$, day1) for 2 to 3 courses followed by a radical hysterectomy. The RR was 78\% [20]. Syoji et al. also reported about NAC followed by a radical hysterectomy for stage of IB2 to IIIB cervical cancer. In this report, 42 patients were treated with intravenous CPT-11 (70 $\mathrm{mg} / \mathrm{m} 2$, day 1 and 8) and cisplatin (70 mg/m2, day1) for two courses followed by a radical hysterectomy. The RR in this report was 83.3\% [24]. Moreover, Syoji et al. also compared neoadjuvant chemotherapy plus radical hysterectomy with radical hysterectomy alone in patients with stage II cervical squamous cell carcinoma presenting as a bulky mass. In this setting, there were no statistically significant differences between the two groups in operative time and the volume of intraoperative blood loss, and the patients in the NAC group were discharge earlier. The hazard ratio for disease-free survival (DFS) in the NAC group, as compared with that in the surgery alone group, was 0.36 (95\% CI 0.08-0.91) [25]. Matsumura et al. reported about NAC follow by a radical hysterectomy 
Table 3: Characteristics of patients with locally invasive cervical cancer who underwent a radical hysterectomy

\begin{tabular}{lccc}
\hline & NAIAC $(\mathbf{n}=\mathbf{8 8})$ & Primary RH $(\mathbf{n}=\mathbf{4 9})$ & p value \\
\hline Age (years old) & $49.0 \pm 11.4$ & $50.5 \pm 11.5$ & \\
FIGO stage & 12 & 15 & 0.04 \\
IB2 & 17 & 17 & 0.1 \\
IIA2 & 59 & 17 & \\
IIB & & & \\
Histology & 68 & 28 & 0.01 \\
Squamous cell carcinoma & 20 & 21 & 0.6 \\
Adenocarcinoma & $45 \pm 12$ & $44 \pm 14$ & \\
Tumor size (mm) & & & 0.07 \\
Adjuvant therapy & 27 & 25 & 0.9 \\
RT or CCRT & 30 & 19 & $<0.01$ \\
Chemotherapy & 31 & 5 & $<0.01$ \\
None & 30 & 48 & 0.02 \\
Median follow up (months) & $64 \%$ & $52 \%$ & \\
3.y. disease free survival & & & \\
\hline
\end{tabular}

NAIAC, neoadjuvant intra-arterial chemotherapy; RH, radical hysterectomy; FIGO, The International Federation of Obstetricians and Gynecologists, RT, radiotherapy; CCRT, concurrent chemoradiotherapy.

for stage of IB2 to IIB cervical cancer. In this report, 48 patients were treated with intravenous CPT-11 $(60 \mathrm{mg} / \mathrm{m} 2$, day 1 and 8$)$ and nedaplatin $(80 \mathrm{mg} / \mathrm{m} 2$, day 1$)$ for 1 to 3 courses followed by a radical hysterectomy. The RR was $75.0 \%$ [23]. Yang et al. also reported about NAC follow by a radical hysterectomy for stage of IB2 to IIB cervical cancer. Among a total of 219 patients, 50 patients received intravenous irinotecan $(60 \mathrm{mg} / \mathrm{m} 2$, day 1,8 and 15) plus cisplatin $(70 \mathrm{mg} / \mathrm{m} 2$, day 1$)$ for two courses followed by a radical hysterectomy, 59 patients received intravenous paclitaxel $(175 \mathrm{mg} / \mathrm{m} 2$, day1) plus cisplatin $(70 \mathrm{mg} / \mathrm{m} 2$, day 1), and 110 patients received primary surgery. The RR in those patients who received irinotecan plus cisplatin was $67.3 \%$, and survival analysis revealed no significant difference in disease-free-survival or OS between the NAC group and the primary surgery group [26]. In these literatures described above, anticancer agents were injected intravenously, and the RR was $67.3-83.3 \%$ [20, 23-26]. In our study, cisplatin was injected intra-arterialy using a $4 \mathrm{~L}-\mathrm{DB}$, and the resulting RR was $92.6 \%$, thus indicating that the RR in our study was higher than that in other previously published literatures. We believe that a high concentrate anticancer drug could be directly delivered to tumors through the uterine artery using a $4 \mathrm{~L}$ $\mathrm{DB}$, thus yielding better results.

There have been several other literatures about intraarterial chemotherapy. Gui et al. reported the comparison of intra-arterial and intravenous NAC in locally advanced cervical cancer. In the study, patients received three cycles of NAC every 3 weeks (cisplatin $70 \mathrm{mg} / \mathrm{m} 2$ on day 1, 5-fluouracil $1000 \mathrm{mg} / \mathrm{m} 2$ on day 1-4). Intra-arterial interventional chemotherapy was administered via right femoral artery catheterization. Each side of the uterine artery received half of the cisplatin dosage. The catheter was retained on the more severe side, maintaining a 24hour infusion of 5-fluouracil for 4 consecutive days. The overall response rate was $84.9 \%$ vs. $88.2 \%$, and the operability rate was $77.4 \%$ vs. $81.4 \%$ for intravenous vs. intra-arterial. There were no significant differences in toxicities, surgical duration, perioperative blood loss, and operative complications between the two groups. The intra-arterial group had a significantly lower parametrial infiltration for postoperative pathological examination. Moreover, the positive vaginal margin, lymph node metastasis and intravascular tumor embolism showed no significant differences. The recurrence, distant metastasis, and 5-year survival rates did not show any significant differences between the two groups [27]. Wen et al. reported a prospective randomized controlled study on multiple neoadjuvant treatment for patients with stage IB2 to IIA cervical cancer. One hundred and twentythree patients were enrolled and randomly assigned to receive one of the following four treatments: radical surgery, brachytherapy with a total dose of $5 \mathrm{~Gy}$ to point A followed by radical surgery, intravenous chemotherapy with cisplatin $50 \mathrm{mg} / \mathrm{m} 2$ plus 5 -fluoroucil $750 \mathrm{mg} / \mathrm{m} 2$ at 
a 2-weeks interval for two courses followed by radical surgery, or intra-arterial chemotherapy with the same regimen as the intravenous chemotherapy followed by radical surgery. The clinical overall response rates were $61.3 \%, 42.9 \%$ and $79.3 \%$ in brachytherapy, intravenous chemotherapy and intra-arterial chemotherapy, respectively. The 3-year progression-free survival rates were $70.7 \%, 66.3 \%, 81.5 \%$ and $79.7 \%$ in surgery alone, brachytherapy, intravenous chemotherapy and intra-arterial chemotherapy, respectively. Three-year overall survival rates were $73.3 \%, 68.3 \%, 82.9 \%$ and $80.4 \%$ in the surgery alone, brachytherapy, intravenous chemotherapy and intraarterial chemotherapy, respectively. Multivariate analysis also showed that only lymph node status correlated with progression-free survival [28]. Tsubamoto et al. reported on neoadjuvant transuterine arterial chemotherapy followed by radical hysterectomy in patients with locally advanced cervical cancer. Seventy-three patients received transuterine arterial chemotherapy combined with intravenous nedaplatin, irinotecan, paclitaxel, or etoposide administration. The radiological response rate was $96 \%$. Multivariate analysis revealed that a tumor size of more than $60 \mathrm{~mm}$ and lymph node metastasis were negative prognostic factors for overall survival. Intra-arterial chemotherapy is still controversial and is not convenient compared to intravenous chemotherapy. However, several literatures have shown that intra-arterial chemotherapy had a higher response rate than the intravenous chemotherapy described above. Moreover, intra-arterial chemotherapy using a 4L-DB could deliver a high concentration of anticancer drug to the tumor - in theory. In our previous report about intra-arterial chemotherapy using a 4L-DB,
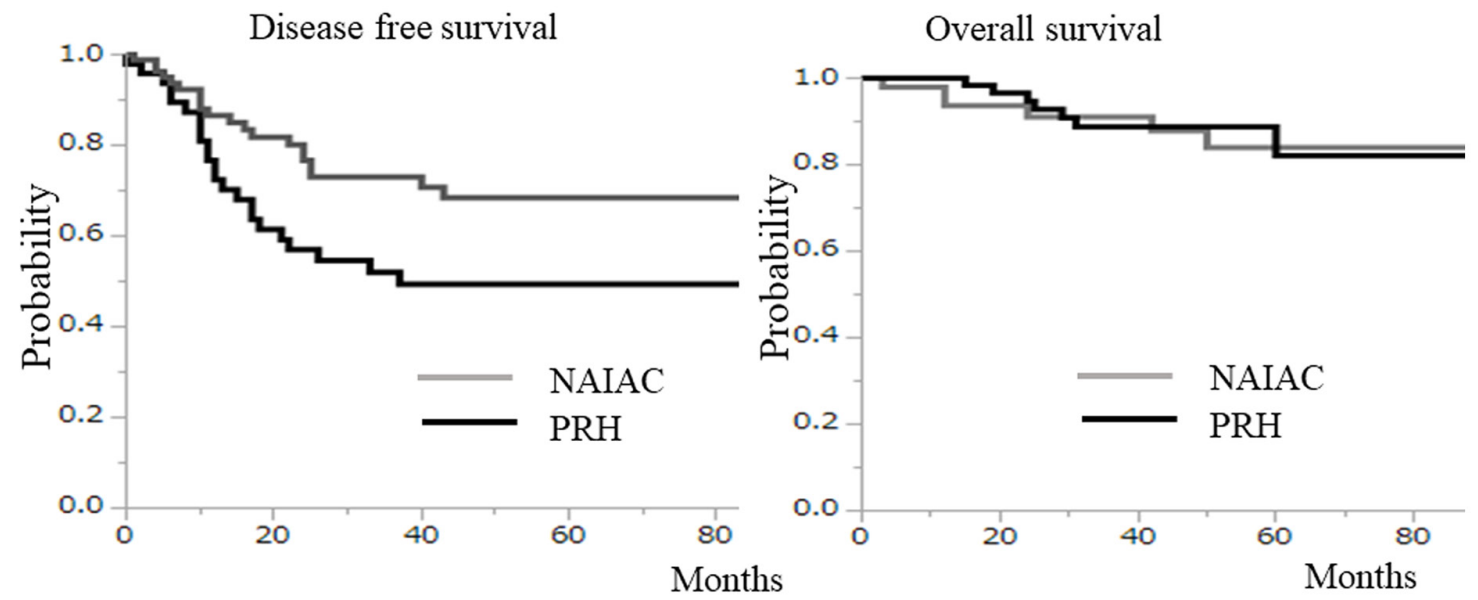

Figure 3: Those patients in the neoadjuvant intraarterial chemotherapy (NAIAC) group had a longer disease free survival rate than those in the primary radical hysterectomy $(\mathbf{P R H})$ group $(\mathbf{p}=\mathbf{0}$. 02). However, overall survival was not signigicantly different between the two groups.

\begin{tabular}{|c|c|c|c|}
\hline \multicolumn{2}{|c|}{ Risk of recurrence } & & \multirow[t]{2}{*}{ Relative risk $(95 \% \mathrm{CI})$} \\
\hline & 0 & & \\
\hline Asdenocarcinoma & & Squamous cell carcinoma & $1.17(0.57-2.31)$ \\
\hline Lymph node metastasis & & No lymph node metastasis & $4.31(2.23-8.43)$ \\
\hline LVI & & No LVI & $1.08(0.47-2.42)$ \\
\hline Deep stromal invasion & & No deep stromal invasion & $0.71(0.32-1.57)$ \\
\hline Bulky tumor & & No bulky tumor & $0.92(0.41-2.07)$ \\
\hline Positive cut end & & Negative cut end & $1.11(0.37-2.66)$ \\
\hline Parametrial invasion & & No parametrial invasion & $0.40(0.14-1.02)$ \\
\hline NAIAC & -1 & $\mathrm{PRH}$ & $0.30(0.14-0.68)$ \\
\hline
\end{tabular}

Figure 4: The results of multivariant analysis for risk of recurrence. Lymph node metastasis and NAIAC were independent factors for recurrence. Other factors including histological type, lymphovascular involvement, deep stromal invasion (more than half myometrial invasion), bulky tumor (more than $4 \mathrm{~cm}$ ), positive cut end, and parametrial invasion were not independent risk factors on multi variant analysis. 
the platinum concentration was significantly higher in the tumors with a CR to NAIAC than those with a PR and SD $(\mathrm{p}<0.0001, \mathrm{CR} ; 11.5 \pm 0.8 \mu \mathrm{g} / \mathrm{g}, \mathrm{PR} ; 7.3 \pm 0.5 \mu \mathrm{g} / \mathrm{g}, \mathrm{SD}$; $4.5 \pm 0.1 \mu \mathrm{g} / \mathrm{g}$ ) [1]. Therefore, intra-arterial chemotherapy, especially via a 4L-DB, may be inconvenient. However, it may have advantages compared with intravenous chemotherapy.

Making comparison between NAC plus radical surgery and primary radiotherapy, a meta-analysis of randomized trials including 872 patients with locally advanced cervical cancer showed that NAC obtained a better DFS $(\mathrm{HR}=0.68,95 \% \mathrm{CI}=0.56-0.82)$ and $\mathrm{OS}(\mathrm{HR}$ $=0.65,95 \% \mathrm{CI}=0.53-0.80$ ) [29] [29]. A recent study by Gupta et al. indicated that cisplatin-based CCRT resulted in superior DFS compared with NAC followed by radical surgery in locally advanced cervical cancer. OS was not significantly different between the two groups. In the subgroup analysis, DFS was significantly inferior in the NAC plus surgery group in stage IIB disease. In stage IB2 and II disease, DFS was not significantly different between the NAC and CCRT groups. However, a lot of patient in this study did not receive complete surgery. $21.5 \%$ of patients crossed over (presurgery crossover and intraoperative unresectable disease) to receive definitive CCRT [30]. In our study, only 6 patients $(6.4 \%)$ receive presurgical CCRT. Furthermore, all patients who underwent radical surgery received complete surgery.

Meta-analysis showed that NAC followed by radical surgery had no prognostic advantage in patients with stage $1 \mathrm{~B} 1$ to IIA cervical cancer, compared to primary radical surgery. NAC was related with a lower rate of tumor size and lymph node metastasis than primary radical surgery. Furthermore, NAC reduced the need for adjuvant radiotherapy and decreased the rate of distant metastasis [11]. The multicenter retrospective study showed that recurrence-free survival was significantly longer in stage IB2 to IIB patients who achieved an overall optimal response to NAC than in those who did not. The authors concluded that optimal responders after NAC followed by radical surgery did not need further treatment. Although this study compared responders with no responders who underwent NAC followed by radical surgery, the sensitivity for NAC may be an important prognostic factor [12]. Other meta-analysis also showed the sensitivity of NAC could be an important prognostic factor in patients with stage IB1 to IV cervical cancer [13]. JCOG0102, which is a prospective randomized control trial for NAC in stage IB1 to IIB cervical cancer in Japan, showed that NAC had no prognostic advantage; however, it did reduce the need for adjuvant radiotherapy [31]. In our study, DFS was significantly longer in those patients who received NAIAC than in those who underwent primary radical surgery. Furthermore, NAIAC was an independent prognostic factor in multivariant analysis, and $35 \%$ patients who received NAIAC did not need any adjuvant therapy. Although further examination is needed, we believe that our 4L-DB could provide excellent results.

This study is associated with several important limitations that may potentially decrease its value. First, the sample size was not large enough for complete analysis. Second, there was selection bias in choosing the primary treatment, as the surgeon tended to choose NAIAC when they felt that it would be difficult to perform a radical hysterectomy due to a large tumor size or parametrial invasion. Third, the results were conducted through those patients who underwent a radical hysterectomy; patients who underwent NAIAC followed by radiotherapy were not considered. Fourth, we did not compare NAIAC plus radical surgery to CCRT. From this point of view, the conclusion is not definitive.

In conclusion, NAIAC using a $4 \mathrm{~L}-\mathrm{DB}$ with intravenous irinotecan and intra-arterial cisplatin is feasible and effective on locally advanced cervical cancer. The NAIAC group had an excellent DFS, as opposed to that in the PRH group. However, overall survival was not significantly different between the groups. The NAIAC group, as well, needed less frequent adjuvant therapy than the PRH group. Therefore, NAIAC using a 4L-DB with intravenous irinotecan and intra-arterial cisplatin followed by radical hysterectomy might be a useful strategy for locally advanced cervical cancer.

\section{MATERIALS AND METHODS}

\section{Participants}

The present study included 143 Japanese patients with cervical cancer who were treated at Osaka Medical College between April 2006 and April 2017. Patients were eligible for inclusion in the study when they met the following criteria: (1) patients with an International Federation of Obstetricians and Gynecologists (FIGO) stage IB2, IIA2 or IIB cervical squamous cell carcinoma or adenocarcinoma who were treated by NAIAC with cisplatin and irinotecan using a 4L-DB or a radical hysterectomy with salpingo-oophorectomy as an initial treatment. The surgeon chose NAIAC when the patients had a seriously restricted uterus for cancer invasion. In contrast, PRH was performed when the patient had no seriously restricted uterus; (2) age less than 70 years; (3) a World Health Organization (WHO) performance status of 0 to 2 ; (4) fulfillment of pretreatment laboratory requirements, including a leukocyte count more than $3000 / \mathrm{mm} 3$, a platelet count more than $100000 / \mathrm{mm} 3$, serum creatinine less than $1.5 \mathrm{mg} / \mathrm{dl}$, serum bilirubin less than $1.5 \mathrm{mg} / \mathrm{dl}$, and normal serum aspartate transaminase (AST) and serum alanine transaminase (ALT); (5) patients without any other major organ disease; and (6) the patient had sufficient clinical data regarding the oncologic outcome, including the date of recurrence. 
All patients were staged according to FIGO criteria, and the histological subtype was assigned according to the criteria of the WHO classification. The present study was approved by the institutional review board (IRB) of Osaka Medical College. Written informed consent was obtained from all patients for NAIAC and for the use of clinical records in the present study. Those patients who underwent a primary radical hysterectomy provided their written informed consent at the time of the primary surgery to use their clinical records for an IRB-approved study, and the IRB approved this consent procedure.

\section{Intra-arterial chemotherapy}

For intra-arterial infusion therapy, we developed an original 4L-DB catheter (Clinical Supply Japan) for the simple and efficient injection of an anticancer agent at a high concentration to target spots in patients with advanced uterine cervical cancer [1]. Under local anesthesia, following Seldinger's technique, polyethylene catheters of 6-French diameter were inserted through both femoral arteries. Each catheter tip was placed in the internal iliac artery. While the guidewire was detained in the peripheral artery of the internal iliac artery, the catheter was passed through the junction of the uterine artery, which was the target vessel, just distal to the branching out of the superior gluteal artery. To confirm the correct position of the catheter and effective perfusion, pelvic arteriography was performed during catheterization procedures. Each time after the completion of treatment, the catheters were removed, and sandbags were used to apply firm pressure over each groin area for $6 \mathrm{~h}$. The regimen included the following: intravenous irinotecan (CPT-11) $(70 \mathrm{mg} / \mathrm{m} 2$, day1 and 8) and intra-arterial cisplatin $(75 \mathrm{mg} / \mathrm{m} 2$, day2) using the 4L-DB for two courses every 21 days. Cisplatin was administered intra-arterially within 30min in divided doses via the bilateral internal iliac arteries. Hydration with normal saline and 5\% dextrose began $3 \mathrm{~h}$ before chemotherapy, with careful monitoring of urine volume.

\section{Treatment response}

Complete blood cell counts and renal and hepatic function tests were repeated before each course. Toxicity was graded according to the WHO criteria. The tumor was monitored with magnetic resonance imaging (MRI; SIGNA MR/i; GE, Slough, UK). Response was measured as the product of the two largest perpendicular diameters of the cervical mass lesion. Patients were evaluated for response with a physical examination and MRI after two courses of therapy. A CR was defined as the complete disappearance of all clinically detectable disease. A 50\% or more decrease in tumor size constituted a partial PR. A SD was defined as no significant change, and a PD was defined as a more than $25 \%$ increase in tumor size or the appearance of new lesions or hydronephrosis.
In addition, histological changes were also evaluated in surgical specimens using the following criteria set by the Japan Society for Cancer Therapy: grade 0 was defined as the absence of degenerative or necrotic change after chemotherapy; grade 1a was defined as degeneration, necrosis, or cytolysis in less than one-third of the cancer cells; grade $1 \mathrm{~b}$ was defined as degeneration, necrosis, or cytolysis in more than one-third but less than two-thirds of the cancer cells; grade 2 was defined as remarkable degeneration, necrosis, cytolysis, or the disappearance of cancer cells in more than two-thirds of the cancer cells; grade 3 was defined as all cancer cells becoming necrotic, the occurrence of cytolysis, or the presence of granulomatous tissue or fibrosis.

\section{Local therapy}

Following NAIAC, a radical hysterectomy with pelvic lymphadenectomy was performed on patients responding to the NAIAC, if possible. After surgery, patients with any poor prognostic factors, including lymph node metastasis, parametrial infiltration, vaginal invasion, or ovarian metastasis received postoperative adjuvant radiotherapy (RT) [Linac, 40 to $50 \mathrm{~Gy}$; and/or RALS (Remote After Loading System), 30 to $40 \mathrm{~Gy}$ ] or chemotherapy. Patients who did not respond to NAIC received CCRT, which was external-beam radiation (Linac, 50-60 Gy) with Cisplatin $40 \mathrm{mg} / \mathrm{m} 2 /$ week and brachytherapy delivered by remote control after loading the system using $60 \mathrm{CO}$ (RALS, 40 to $50 \mathrm{~Gy}$ ).

\section{Statistical analysis}

All of the statistical analyses were performed using the JMP software package (version. 11.1.1). Continuous variables are expressed as the mean \pm standard deviation. The Student T-test was used to compare continuous variables, and Pearson's Chi-square test was used to compare frequencies. Overall survival curves and DFS curves were plotted according to the Kaplan-Meier method and were analyzed by the log-rank test. Relative risk was calculated by Cox's proportional hazards model. $P$ values of $<0.05$ were considered to indicate statistical significance.

\section{Abbreviations}

4L-DB: four-lumen double-balloon catheter; NAIAC: neoadjuvant intra-arterial chemotherapy; PRH: primary radical hysterectomy; RH: radical hysterectomy; RR: relative risk; NCCN: National Comprehensive Cancer Network's; NAC: neoadjuvant chemotherapy; CCRT: concurrent chemoradiotherapy; RT: radiotherapy; SD: standard deviation; FIGO: International Federation of Obstetricians and Gynecologists; CR: complete response; PR: partial response; SD: stable disease; PD: progressive disease; DFS: disease-free survival. 


\section{Author contributions}

T.T., and M.O. designed study; T.T., Y.Te., S.F., Y.Ta. and H.S. performed data collection and analysis; T.T., Y.Te, Y.Ta., and T.Y. made diagnosis of histological analyses; T.T., S.T., and K.Y. made diagnosis of image analyses; K.Y performed NAIAC; T.T., and M.O. wrote the paper.

\section{ACKNOWLEDGMENTS}

Mr. Niinobe Shigefumi (Osaka Medical College Biomedical Computation Center) attributed to the artwork design.

\section{CONFLICTS OF INTEREST} interest.

The authors declare that they have no conflicts of

\section{FUNDING}

The authors have received no funding for this article.

\section{REFERENCES}

1. Terai Y, Kanemura M, Sasaki H, Tsunetoh S, Tanaka Y, Yamashita Y, Yamamoto K, Narabayashi I, Ohmichi M. Long-term follow-up of neoadjuvant intraarterial chemotherapy using an original four-lumen double-balloon (4L-DB) catheter for locally advanced uterine cervical cancer. Int J Clin Oncol. 2009; 14:56-62. https://doi. org/10.1007/s10147-008-0801-3.

2. Azuma H, Inamoto T, Ibuki N, Ubai T, Kotake Y, Takahara K, Kiyama S, Nomi H, Uehara H, Komura K, Yamamoto K, Narumi Y, Katsuoka Y. Novel bladder preservation therapy for locally invasive bladder cancer: combined therapy using balloon-occluded arterial infusion of anticancer agent and hemodialysis with concurrent radiation. Int J Oncol. 2010; 37:773-85.

3. Azuma H, Inamoto T, Ibuki N, Ubai T, Kotake Y, Takahara K, Kiyama S, Nomi H, Uehara H, Komura K, Yamamoto K, Narumi Y, Katsuoka Y. Utility of the novel bladder preservation therapy, BOAI-CDDP-radiation (OMCregimen), for elderly patients with invasive bladder cancer. Int J Oncol. 2011; 38:13-24.

4. Azuma $\mathrm{H}$, Inamoto $\mathrm{T}$, Takahara $\mathrm{K}$, Nomi $\mathrm{H}$, Hirano $\mathrm{H}$, Ibuki N, Uehara H, Komura K, Minami K, Uchimoto T, Saito K, Takai T, Tanda N, et al. The novel bladder preservation therapy BOAI-CDDP-radiation (OMCregimen): a new treatment option for invasive bladder cancer patients with lymph node metastasis. Int J Oncol. 2014; 44:1895-903. https://doi.org/10.3892/ ijo.2014.2378.
5. Azuma H, Inamoto $\mathrm{T}$, Takahara $\mathrm{K}$, Nomi H, Hirano $\mathrm{H}$, Uehara H, Komura K, Minami K, Kouno J, Kotake Y, Abe H, Takagi S, Ibuki N, et al. A great option for elderly patients with locally invasive bladder cancer, BOAI-CDDPradiation (OMC regimen). Int J Oncol. 2013; 43:1087-94. https://doi.org/10.3892/ijo.2013.2058.

6. Azuma $\mathrm{H}$, Inamoto $\mathrm{T}$, Takahara $\mathrm{K}$, Nomi $\mathrm{H}$, Uehara H, Komura K, Minami K, Kouno J, Kotake Y, Abe H, Takagi S, Yamamoto K, Narumi Y, et al. Effect of a novel bladder preservation therapy, BOAI-CDDP-radiation (OMC-regimen). Int J Oncol. 2013; 43:79-87. https://doi. org/10.3892/ijo.2013.1923.

7. Azuma H, Kotake Y, Yamamoto K, Sakamoto T, Kiyama $\mathrm{S}$, Ubai T, Inamoto T, Takahara K, Matsuki M, Segawa N, Shibahara N, Katsuoka Y. Effect of combined therapy using balloon-occluded arterial infusion of cisplatin and hemodialysis with concurrent radiation for locally invasive bladder cancer. Am J Clin Oncol. 2008; 31:11-21. https:// doi.org/10.1097/COC.0b013e318136e27a.

8. Azuma H, Yamamoto K, Inamoto T, Ibuki N, Kotake Y, Sakamoto T, Kiyama S, Ubai T, Takahara K, Segawa N, Narumi Y, Katsuoka Y. Total cystectomy versus bladder preservation therapy for locally invasive bladder cancer: effect of combined therapy using balloon-occluded arterial infusion of anticancer agent and hemodialysis with concurrent radiation. Am J Clin Oncol. 2009; 32:592-606. https://doi.org/10.1097/COC.0b013e318199fb42.

9. Landoni F, Maneo A, Colombo A, Placa F, Milani R, Perego P, Favini G, Ferri L, Mangioni C. Randomised study of radical surgery versus radiotherapy for stage Ib-IIa cervical cancer. Lancet. 1997; 350:535-40. https://doi.org/10.1016/ s0140-6736(97)02250-2.

10. National Comprehensive Cancer Network. Cervical Cancer (Version 1.2018).

11. Kim HS, Sardi JE, Katsumata N, Ryu HS, Nam JH, Chung HH, Park NH, Song YS, Behtash N, Kamura T, Cai HB, Kim JW. Efficacy of neoadjuvant chemotherapy in patients with FIGO stage IB1 to IIA cervical cancer: an international collaborative meta-analysis. Eur J Surg Oncol. 2013; 39:115-24. https://doi.org/10.1016/j.ejso.2012.09.003.

12. Landoni F, Sartori E, Maggino T, Zola P, Zanagnolo V, Cosio S, Ferrari F, Piovano E, Gadducci A. Is there a role for postoperative treatment in patients with stage Ib2-IIb cervical cancer treated with neo-adjuvant chemotherapy and radical surgery? An Italian multicenter retrospective study. Gynecol Oncol. 2014; 132:611-7. https://doi.org/10.1016/j. ygyno.2013.12.010.

13. Ye Q, Yuan HX, Chen HL. Responsiveness of neoadjuvant chemotherapy before surgery predicts favorable prognosis for cervical cancer patients: a meta-analysis. J Cancer Res Clin Oncol. 2013; 139:1887-98. https://doi.org/10.1007/ s00432-013-1509-y.

14. Young RH, Clement PB. Endocervical adenocarcinoma and its variants: their morphology and differential diagnosis. Histopathology. 2002; 41:185-207. 
15. Eifel PJ, Morris M, Oswald MJ, Wharton JT, Delclos L. Adenocarcinoma of the uterine cervix. Prognosis and patterns of failure in 367 cases. Cancer. 1990; 65:2507-14.

16. Lai CH, Hsueh S, Hong JH, Chang TC, Tseng CJ, Chou $\mathrm{HH}$, Huang KG, Lin JD. Are adenocarcinomas and adenosquamous carcinomas different from squamous carcinomas in stage IB and II cervical cancer patients undergoing primary radical surgery? Int J Gynecol Cancer. 1999; 9:28-36.

17. Shingleton HM, Bell MC, Fremgen A, Chmiel JS, Russell $\mathrm{AH}$, Jones WB, Winchester DP, Clive RE. Is there really a difference in survival of women with squamous cell carcinoma, adenocarcinoma, and adenosquamous cell carcinoma of the cervix? Cancer. 1995; 76:1948-55.

18. Thigpen T. The role of chemotherapy in the management of carcinoma of the cervix. Cancer J. 2003; 9:425-32.

19. Tsuda H, Hashiguchi Y, Nishimura S, Miyama M, Nakata S, Kawamura N, Negoro S. Phase I-II study of irinotecan (CPT-11) plus nedaplatin (254-S) with recombinant human granulocyte colony-stimulating factor support in patients with advanced or recurrent cervical cancer. Br J Cancer. 2004; 91:1032-7. https://doi.org/10.1038/sj.bjc.6602076.

20. Sugiyama T, Nishida T, Kumagai S, Nishio S, Fujiyoshi K, Okura N, Yakushiji M, Hiura M, Umesaki N. Combination therapy with irinotecan and cisplatin as neoadjuvant chemotherapy in locally advanced cervical cancer. Br J Cancer. 1999; 81:95-8. https://doi.org/10.1038/ sj.bjc. 6690656.

21. Shoji T, Takatori E, Hatayama S, Omi H, Kagabu M, Honda T, Kumagai S, Morohara Y, Miura F, Yoshizaki A, Sugiyama T. Phase II study of tri-weekly cisplatin and irinotecan as neoadjuvant chemotherapy for locally advanced cervical cancer. Oncol Lett. 2010; 1:515-9. https://doi.org/10.3892/ ol_00000091.

22. Abou-Taleb HA, Koshiyama M, Matsumura N, Baba T, Yamaguchi K, Hamanishi J, Abiko K, Yamanoi K, Murakami R, Horikawa N, Taha AA, Kitamura S, Konishi I. Clinical efficacy of neoadjuvant chemotherapy with irinotecan (CPT-11) and nedaplatin followed by radical hysterectomy for locally advanced cervical cancer. J Int Med Res. 2016; 44:346-56. https://doi. org/10.1177/0300060515591858.

23. Matsumura M, Takeshima N, Ota T, Omatsu K, Sakamoto K, Kawamata Y, Umayahara K, Tanaka H, Akiyama F, Takizawa K. Neoadjuvant chemotherapy followed by radical hysterectomy plus postoperative chemotherapy but no radiotherapy for Stage IB2-IIB cervical cancer-irinotecan and platinum chemotherapy. Gynecol Oncol. 2010; 119:212-6. https://doi.org/10.1016/j. ygyno.2010.07.031.

24. Shoji T, Takatori E, Furutake Y, Takada A, Nagasawa T, Omi H, Kagabu M, Honda T, Miura F, Takeuchi S, Kumagai
S, Yoshizaki A, Sato A, et al. Phase II clinical study of neoadjuvant chemotherapy with CDDP/CPT-11 regimen in combination with radical hysterectomy for cervical cancer with a bulky mass. Int J Clin Oncol. 2016; 21:1120-7. https://doi.org/10.1007/s10147-016-1008-7.

25. Takatori E, Shoji T, Takada A, Nagasawa T, Omi H, Kagabu M, Honda T, Miura F, Takeuchi S, Sugiyama T. A retrospective study of neoadjuvant chemotherapy plus radical hysterectomy versus radical hysterectomy alone in patients with stage II cervical squamous cell carcinoma presenting as a bulky mass. Onco Targets Ther. 2016; 9:5651-7. https://doi.org/10.2147/ott.s101146.

26. Yang Z, Chen D, Zhang J, Yao D, Gao K, Wang H, Liu $\mathrm{C}, \mathrm{Yu} \mathrm{J}, \mathrm{Li}$ L. The efficacy and safety of neoadjuvant chemotherapy in the treatment of locally advanced cervical cancer: A randomized multicenter study. Gynecol Oncol. 2016; 141:231-9. https://doi.org/10.1016/j. ygyno.2015.06.027.

27. Gui T, Shen K, Xiang Y, Pan L, Lang J, Wu M, Huang H, Cao D, Yang J. Neoadjuvant chemotherapy in locally advanced cervical carcinoma: which is better, intravenous or intra-arterial? Onco Targets Ther. 2014; 7:2155-60. https://doi.org/10.2147/ott.s67633.

28. Wen H, Wu X, Li Z, Wang H, Zang R, Sun M, Huang $\mathrm{X}$, Zhang Z, Cai S. A prospective randomized controlled study on multiple neoadjuvant treatments for patients with stage IB2 to IIA cervical cancer. Int J Gynecol Cancer. 2012; 22:296-302. https://doi.org/10.1097/ IGC.0b013e31823610a1.

29. Hu T, Li S, Chen Y, Shen J, Li X, Huang K, Yang R, Wu L, Chen Z, Jia Y, Wang S, Cheng X, Han X, et al. Matchedcase comparison of neoadjuvant chemotherapy in patients with FIGO stage IB1-IIB cervical cancer to establish selection criteria. Eur J Cancer. 2012; 48:2353-60. https:// doi.org/10.1016/j.ejca.2012.03.015.

30. Gupta S, Maheshwari A, Parab P, Mahantshetty U, Hawaldar R, Sastri Chopra S, Kerkar R, Engineer R, Tongaonkar H, Ghosh J, Gulia S, Kumar N, Shylasree TS, et al. Neoadjuvant Chemotherapy Followed by Radical Surgery Versus Concomitant Chemotherapy and Radiotherapy in Patients With Stage IB2, IIA, or IIB Squamous Cervical Cancer: A Randomized Controlled Trial. J Clin Oncol. 2018; 36:1548-55. https://doi. org/10.1200/jco.2017.75.9985.

31. Katsumata N, Yoshikawa H, Kobayashi H, Saito T, Kuzuya K, Nakanishi T, Yasugi T, Yaegashi N, Yokota H, Kodama S, Mizunoe T, Hiura M, Kasamatsu T, et al. Phase III randomised controlled trial of neoadjuvant chemotherapy plus radical surgery vs radical surgery alone for stages IB2, IIA2, and IIB cervical cancer: a Japan Clinical Oncology Group trial (JCOG 0102). Br J Cancer. 2013; 108:1957-63. https://doi.org/10.1038/bjc.2013.179. 